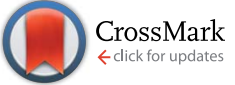

Cite this: Chem. Sci., 2016, 7, 2359

\title{
Multi-luminescent switching of metal-free organic phosphors for luminometric detection of organic solvents $\dagger$
}

\begin{abstract}
Min Sang Kwon, ${ }^{a}$ Jake H. Jordahl, ${ }^{b}$ Andrew W. Phillips, ${ }^{c}$ Kyeongwoon Chung, ${ }^{c}$ Sunjong Lee, ${ }^{f}$ Johannes Gierschner, ${ }^{\text {g Joerg Lahann }}{ }^{\text {abcd }}$ and Jinsang Kim ${ }^{\star a b c d e}$

Metal-free organic phosphors can be an attractive smart optical sensing materials since, in such compounds, intersystem crossing (ISC) and the phosphorescence process are placed in subtle competition with fluorescence, internal conversion (IC), and non-radiative decay pathways. Here, we report a unique environment-dependent multi-luminescence switching behavior of metal-free organic phosphorescent materials. Through combined photophysical measurements and computational electronic structure analysis, we systematically investigated how physicochemical properties of organic solvents affect the photophysical pathways of the metal-free organic phosphors. By rationally adapting the finding into phosphor-doped electrospun polymer fibers, we developed a new luminometric sensory platform and achieved selective detection of eight different common organic solvents. The presented finding provides new possibilities for metal-free organic phosphors to be a novel class of smart optical sensory materials.
\end{abstract}

Received 20th October 2015

Accepted 25th December 2015

DOI: $10.1039 / \mathrm{c} 5 \mathrm{sc03986j}$

www.rsc.org/chemicalscience stacking and/or conformation. Although much effort has been devoted to these material classes, development of a new material class having novel luminescent transduction mechanisms can significantly improve the desired properties and extend the potential application of smart luminescent materials.

Metal-free organic phosphorescent materials are photophysically distinct from conventional fluorescent materials and phosphorescent organometallic complexes. Only certain classes of organic emitters having particular moieties (for example, aromatic carbonyls, nitrogen heterocycles, and aromatic sulfides) $^{6}$ show room temperature phosphorescence (RTP) because spin-orbit coupling (SOC) is typically inefficient without heavy-metal atom. In such compounds, intersystem crossing (ISC) from $\mathrm{S}_{1}$ to $\mathrm{T}_{n}$ and the phosphorescence process is not fast enough $\left(k_{\mathrm{P}}<10^{2} \mathrm{~s}^{-1}\right)$ and, thereby, those processes are placed in subtle competition with fluorescence, internal conversion (IC), and non-radiative decay pathways (Scheme 1b). Therefore, the emission properties of metal-free organic phosphors are sensitively dependent on (1) the nature of excited electronic states (i.e. the nature of $\mathrm{S}_{1}, \mathrm{~T}_{n}$, and $\mathrm{T}_{1}$ ) and (2) radiationless deactivation pathways, which are susceptible to environmental conditions like the presence of oxygen, rigidity of matrices, and temperature. ${ }^{7}$ Despite the fact that these photophysical properties make metal-free organic phosphors attractive smart optical sensing materials, metal-free organic phosphorescent materials have been rarely explored for sensing except for recent oxygen sensors. ${ }^{\mathbf{8}, 9 e}$

Here, we report environment-responsive multi-luminescence switching behavior of metal-free organic phosphorescent 
a)

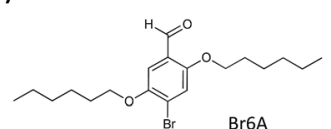

materials. The phosphors described in Scheme 1a, Br6A and G1, exhibit strong green phosphorescence in specific polymer matrices while they show either blue fluorescence or no luminescence in solutions depending on solvent polarity (Scheme 1c). Through combined experimental analysis and quantum chemical (QC) calculations, we thoroughly investigate the photophysical pathways of metal-free organic phosphors to understand the observed multi-luminescence switching. Moreover, we develop a new sensory platform for luminometric detection of organic solvents based on the multi-luminescence switching behavior of the phosphors. Combinations of three different types of polymer composite fiber mats produce unique luminescence color patterns, which makes it possible to successfully differentiate eight different common organic solvents.

\section{Results and discussions}

The phosphors, Br6A and G1, show strong green RTP in specific amorphous polymer matrices, i.e. isotactic poly(methyl methacrylate) (iPMMA) and poly(vinyl alcohol) (PVA), at ambient conditions (Scheme 1c). As we reported previously, this is due to the combined characteristic molecular features of the phosphors and of the host polymer matrices. ${ }^{9}$ The phosphors promote ISC via SOC by the creation of a $n \pi^{*}$ type triplet $\left(\mathrm{T}_{\mathrm{n}}\right)$ state through the benzaldehyde moiety as well as by the intraand intermolecular heavy atom effect of bromine through direct participation in the relevant frontier $\pi / \pi^{*}$-type MOs, providing efficient solid-state phosphorescence (see below for details). The rigidity, high triplet energy, and oxygen barrier properties of host polymer matrices can greatly suppress the radiationless deactivation processes of triplet excitons, resulting in efficient RTP in host-guest polymer composites (Table S1†). ${ }^{8-10}$

The phosphors (Br6A and G1) also show interesting solventdependent photophysical properties in solution. In polar solvents, e.g. methanol (MeOH) and water, the molecules show strong blue fluorescence. In other solvents like chloroform $\left(\mathrm{CHCl}_{3}\right)$, fluorescence is effectively switched off (Fig. 1). No
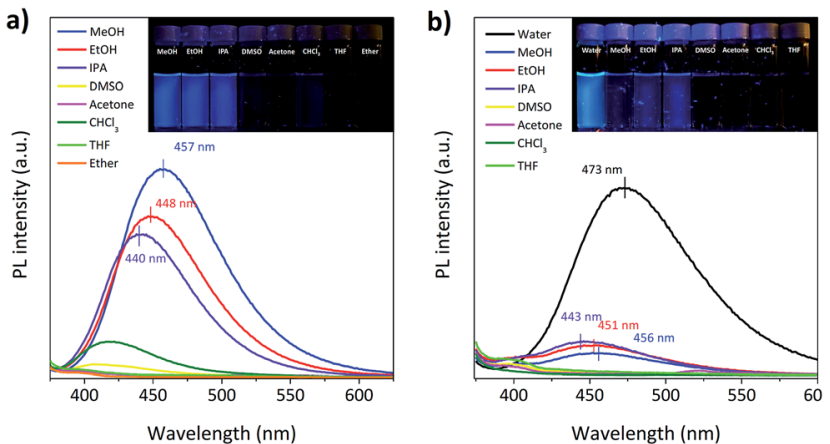

Fig. 1 Fluorescence spectra of (a) Br6A and (b) G1 in various solvents are presented. Inset shows the fluorescence images of luminophores in solution under a $365 \mathrm{~nm}$ handheld UV lamp.

phosphorescence is observed under gated conditions at room temperature in their deoxygenated solutions. On the other hand, cooling the phosphor solutions to $77 \mathrm{~K}$ or introducing them into solid matrices of iPMMA or PVA, result in noticeable phosphorescence (Scheme 1c and Table S1 $\dagger$ ). ${ }^{9}$ Photophysical analysis, combined with time-dependent density functional theory (TD-DFT) calculations, were conducted to elucidate the process. ${ }^{11}$ Chloroform solution of Br6A showed detectable fluorescence so that we could quantify fluorescence quantum efficiency (QY) and lifetime and we thus successfully compared with the values in methanol solution of Br6A (Table 1), allowing for the quantitative analysis of the observed multi-luminescence switching. However, unfortunately, it is technically very difficult to quantify these values in some non-polar solvents such as ether since the molecules are barely fluorescent as one can see in Fig. 1.

The main characteristics of the photophysical processes were analyzed by UV-vis absorption and steady-state and timeresolved photoluminescence (PL) spectroscopy (Table 1 and Fig. 1 and $\mathrm{S} 1$ in the $\mathrm{ESI} \dagger)$. In $\mathrm{MeOH}$, the radiative rate $\left(k_{\mathrm{F}}\right)$ from $\mathrm{S}_{1}$ to $\mathrm{S}_{0}$ is $0.053 \mathrm{~ns}^{-1}$, while in $\mathrm{CHCl}_{3}$ it is only $0.010 \mathrm{~ns}^{-1}$. Thus, the oscillator strength of the emitting state $(f)$ must be significantly different in the two solvents. The oscillation strength is accessible via the Strickler-Berg equation, ${ }^{\mathbf{1 2 , 1 3}}$ which relates $f$ with $k_{\mathrm{F}}$, giving $f_{\mathrm{MeOH}}=0.11$, whereas $f_{\mathrm{CHCl}_{3}}=0.017$. On the other hand, $f$ of the main absorption extracted by integration of the absorption spectrum is $f=0.09$ (Table 1 ). This compares well with the photophysical data in $\mathrm{MeOH}$, and thus indicates that in this case emission originates from an allowed transition from $S_{0}$ to $S_{1}$ which gives rise to the main absorption. Contrary, in $\mathrm{CHCl}_{3}$, an essentially dark $\mathrm{S}_{1}$ state has to be proposed. Thus, from the experimental data we suggest a solvent-dependent stateswitching mechanism.

The alternative deactivation pathways for fluorescence $\left(k_{\mathrm{nF}}\right)$ are internal conversion $\left(k_{\mathrm{IC}}\right)$ and ISC $\left(k_{\mathrm{ISC}}\right.$ to the triplet manifold with consecutive phosphorescence, $k_{\mathrm{P}}$, or ISC to $\mathrm{S}_{0}, k_{\mathrm{TS}}$, or quenching processes to $\left.\mathrm{S}_{0}, k_{\mathrm{q}}\right)$; $k_{\mathrm{nF}}$ is considerably slower by a factor of six in $\mathrm{MeOH}\left(0.363 \mathrm{~ns}^{-1}\right)$ compared to $\mathrm{CHCl}_{3}(1.990$ $\left.\mathrm{ns}^{-1}\right)$, see Table 1 . Considering that no phosphorescence is observed at room temperature in neither solvents, at least one of the radiationless deactivation pathways $\left(k_{\mathrm{IC}}, k_{\mathrm{ISC}}, k_{\mathrm{TS}}\right.$, 
Table 1 Photophysical data of Br6A. Measured absorption and fluorescence maxima, fluorescence quantum yields $\left(\Phi_{\mathrm{F}}\right)$ and lifetimes $\left(\tau_{\mathrm{F}}\right)$, rate constants for radiative $\left(k_{\mathrm{F}}\right)$ and radiationless deactivation $\left(k_{\mathrm{nF}}\right)$, extracted via $\Phi_{\mathrm{F}}=k_{\mathrm{F}} \tau_{\mathrm{F}}=k_{\mathrm{F}} /\left(k_{\mathrm{F}}+k_{\mathrm{nF}}\right)$, where $k_{\mathrm{nF}}$ includes both, internal conversion $\left(k_{\mathrm{IC}}\right)$ and intersystem crossing $\left(k_{\mathrm{ISC}}\right)$. Oscillator strength $(f)$ extracted from the absorption spectrum, via the Strickler-Berg relation and from TDDFT

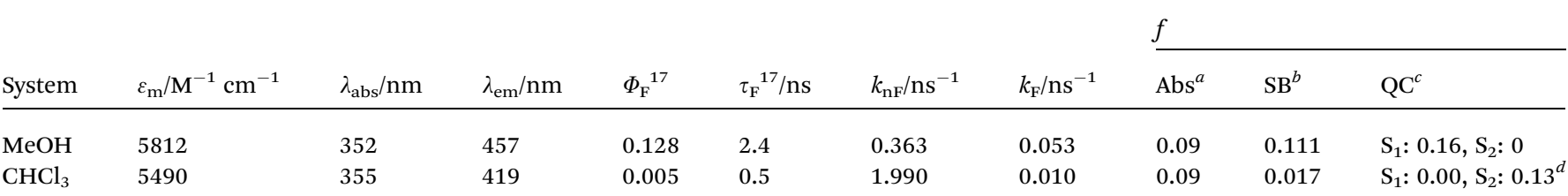

${ }^{a}$ From $f=4.319 \times 10^{-9}\left[\mathrm{M}^{-1} \mathrm{~cm}^{2}\right] \int \varepsilon_{\mathrm{m}}(\nu) \mathrm{d} \nu .{ }^{b}$ From the simplified Strickler-Berg relation. ${ }^{12,13}{ }^{c}$ From QC calculations (TD-DFT). ${ }^{d}$ In vacuum.

$k_{\mathrm{q}}$ ), must be very fast relative to $k_{\mathrm{F}}$ under these conditions (Scheme 1a).

In order to understand the solvent-dependent state-switching and the impact on radiationless deactivation, we performed TDDFT calculations. ${ }^{14}$ In vacuum, the $\mathrm{S}_{1}$ state, calculated at $3.66 \mathrm{eV}$ ( $339 \mathrm{~nm}$ ), is of $n \pi^{*}$ nature (Fig. 2 and Table S2 in the ESI $\dagger$ ) and is thus forbidden $(f=0),{ }^{15}$ whereas the bright $\mathrm{S}_{2}$ state of $\pi \pi^{*}$ nature state is calculated at $3.77 \mathrm{eV}(329 \mathrm{~nm})$ with $f=0.13$. In $\mathrm{MeOH}$, the $\mathrm{n} \pi^{*}$ character is essentially unchanged $(3.78 \mathrm{eV} ; 328$ $\mathrm{nm}$ ), while the $\pi \pi^{*}$ state is significantly stabilized ( $3.61 \mathrm{eV} ; 343$ $\mathrm{nm}$ ) and becomes the emissive state, which is in agreement with experiment. It should be noted, however, that although TDDFT accurately predicts the qualitative behavior, i.e. the stateswitching by a solvent effect, it does not capture all details. In fact, state-switching is predicted already for $\mathrm{CHCl}_{3} \cdot{ }^{16}$

Radiationless processes cannot be drawn from QC calculations as long as the excited state hypersurfaces are not known in detail, which are not properly accessible with TD-DFT. However, some basic statements can be made. Firstly, the decrease in $k_{\mathrm{nF}}$ in polar $v s$. non-polar solvents cannot be due to ISC from $\mathrm{T}_{1}$ to
$S_{0}$ because the nature of $T_{1}$ is the same in both cases, and the relative change in the state energy is small (Table 1 and Fig. 2b). Although a solvent-dependent change of the IC pathway $\left(\mathrm{S}_{1} \rightarrow\right.$ $S_{0}$ ) cannot be excluded since the nature of $S_{1}$ changes, the peculiar solvent-dependent state ordering suggests an alternative explanation. In $\mathrm{MeOH}$, ISC from $\mathrm{S}_{1}\left(\pi \pi^{*}\right)$ to the energetically close $\mathrm{T}_{3}\left(\pi \pi^{*}\right)$ state is El-Sayed forbidden and is only promoted by SOC through the heavy-atom effect of bromine, while ISC in vacuum is El-Sayed allowed, i.e. a transition from $\mathrm{S}_{1}\left(\mathrm{n} \pi^{*}\right)$ to $\mathrm{T}_{3}\left(\pi \pi^{*}\right)$. The $\mathrm{T}_{2}\left(\mathrm{n} \pi^{*}\right)$ state on the other hand, which could serve for an El-Sayed allowed ISC process in $\mathrm{MeOH}$, is significantly lower in energy and is thus much less efficient for ISC compared to the situation in vacuum. Finally, the phosphorescence process in Br6A is only promoted by the heavy atom effect, and is not El-Sayed allowed, e.g. as in benzophenone. ${ }^{5,6}$ Thus, alternative deactivation pathways, i.e. ISC and quenching processes from $T_{1}$ to $S_{0}$, can effectively compete with phosphorescence at ambient conditions (Scheme 1a and Fig. 2b).

We prepared polymer composites to study the feasibility of an array-based selective detection of common organic solvents.

\section{b)}

a)

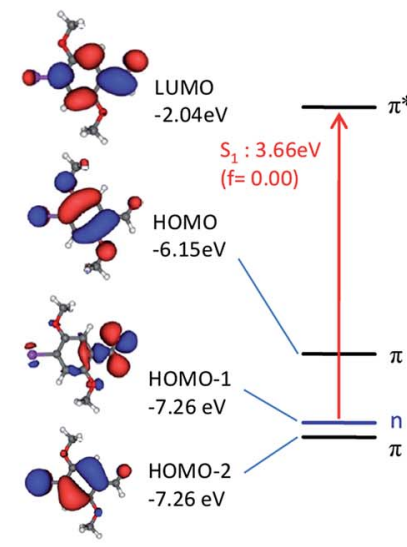

Vacuum (non-polar)

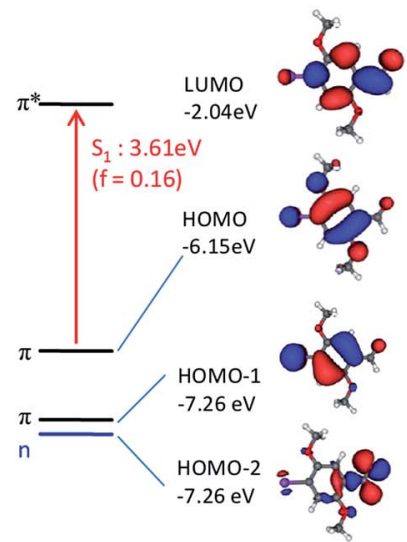

$\mathrm{MeOH}$ (polar)

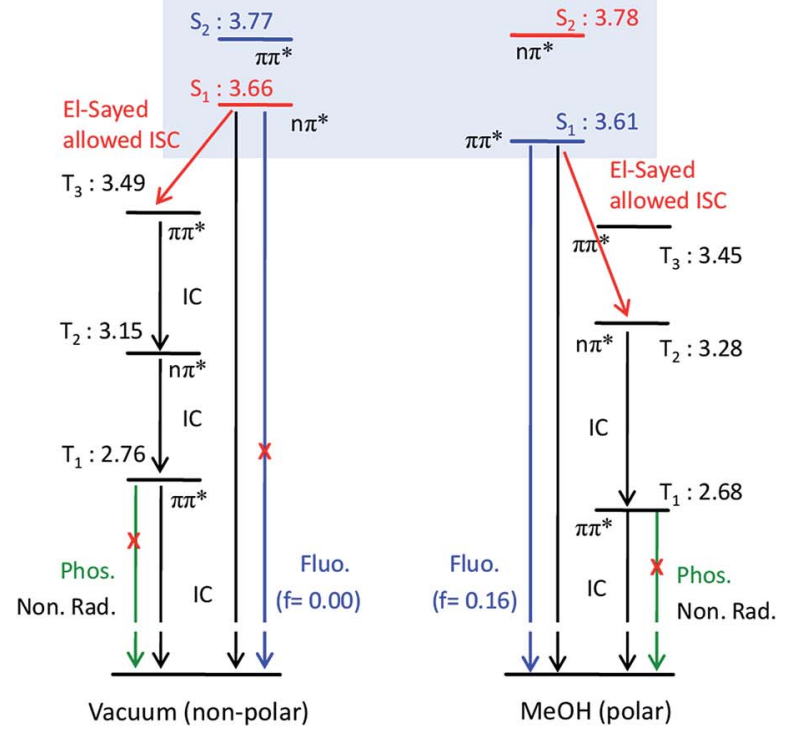

Fig. 2 (a) Equilibrium structure and frontier MOs of Br6A. (b) Term diagrams of Br6A in vacuum and methanol as calculated by TD-DFT; n $\pi$ *-type states are indicated, while the other states are all of $\pi \pi^{*}$ character. Energies are given in $\mathrm{eV}$. 
Array-based detection is a potentially powerful method for the detection of various chemical analytes. ${ }^{18,19}$ Since sensor arrays produce a distinct pattern of responses based on cross-reactive sensor elements, they may provide a characteristic fingerprint for analytes having similar chemical and physical properties. We selected Br6A-iPMMA, G1-PVA80, and G1-PVA100 polymer composites as cross-reactive sensor elements (Fig. 1a and 3). Phosphors act as a signaling unit in the detection platform due to their environment-dependent multi-luminescence. Polymer matrices offer a rigid environment for efficient solid-state phosphorescence of embedded phosphors, serve as an oxygen barrier, and confer additional selectivity through distinct solubility toward different organic solvents. Once the embedded phosphors are exposed to a solvent, they can undergo a distinct luminometric transition: (1) maintain green phosphorescence, (2) change from green phosphorescence to blue fluorescence or (3) become non-luminescent. To prepare polymer composites, electro-spinning was chosen because it allows rapid and costeffective fabrication of fibrous polymer composites with large surface areas, resulting in fast and reproducible differentiation of solvents.

Electrospun fiber mats were fabricated from three different viscous polymer solutions containing the phosphors, Br6A or G1. Polymer solutions were pumped through a syringe needle charged to a high voltage. The resultant microfibers were collected on a high speed rotating drum collector covered with aluminum foil. Scanning electron microscope (SEM) images confirm the successful formation of microfibers by the electrospinning method (Fig. 3a). The detailed fabrication conditions of the polymer composite fibers are given in the ESI. $\dagger$

The resulting composite fiber mats show strong green phosphorescence at ambient conditions, which corresponds

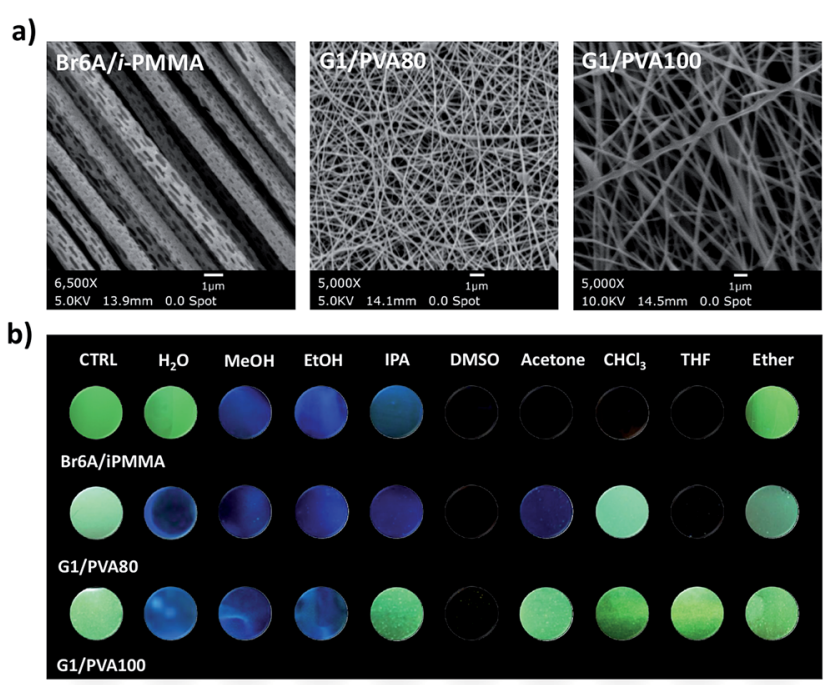

Fig. 3 (a) SEM images of the Br6A-iPMMA (upper), G1-PVA80 (middle), and G1-PVA100 electrospun polymer fiber mats. (b) Photographs of the Br6A-iPMMA (upper), G1-PVA80 (middle), and G1PVA100 electrospun polymer fiber mats before (CTRL) and after exposure to various organic solvents under a $365 \mathrm{~nm}$ hand-held UV lamp. well with conventional drop-cast films in terms of emission wavelength and phosphorescence intensity (Fig. 3b). This implies that electrospinning is an efficient method to make phosphorescent polymer fiber composites. Pipette drops ( $c a .50$ $\mu \mathrm{l})$ of organic solvents were placed onto the three different fiber mats and the resulting luminescence color change was monitored after $5 \mathrm{~min}$. The polymer mats show distinctively different luminometric responses upon exposure to the organic solvents. For example, the Br6A-iPMMA composite polymer mats displayed the green phosphorescence to blue fluorescence transition when it was exposed to polar solvents like $\mathrm{MeOH}$, ethanol (EtOH), and isopropanol (IPA). Alcoholic solvents can dissolve the polymer matrix and therefore efficiently break the halogen bonded luminophore networks, resulting in phosphorescence quenching and blue fluorescence emission, like in solution. Solvents with medium polarity such as dimethyl sulfoxide (DMSO), acetone, $\mathrm{CHCl}_{3}$, and tetrahydrofuran (THF) can also dissolve the polymer matrix and thus induce the transition from green phosphorescence to non-luminescence. Since water and ether cannot dissolve the polymer matrix, the polymer mats maintain their original green phosphorescence emission properties. G1-PVA80 and G1-PVA100 also show their own characteristic luminescence patterns in response to solvents because the polymers have different solubility parameters in the solvents. THF and IPA dissolved PVA80 well and, thus, phosphorescence was efficiently quenched, while phosphorescence was maintained in PVA100 for both solvents. Based on the combination/patterns of responses, we successfully differentiated eight different solvents by simply monitoring the emission color patterns.

More interestingly, $\mathrm{MeOH}$ and $\mathrm{EtOH}$, which show exactly the same emission color patterns, were further distinguished by response time. Much faster emission color change (from green phosphorescence to blue fluorescence) was observed in $\mathrm{MeOH}$ compared to EtOH (Movie S1 in the ESI $\dagger$ ). We attribute this difference in response time to the different solubility of iPMMA for $\mathrm{MeOH}$ and EtOH.

\section{Conclusions}

In conclusion, we report a unique environment-dependent multi-luminescence switching of metal-free organic phosphorescent materials by controlling competing photophysical pathways. Through combined photophysical and electronic structure analysis, we reveal that the observed multi-luminescence switching is due to subtle changes in the photophysical pathways of the metal-free organic phosphors, e.g. solventdependent state-switching. By rationally adapting the finding, we developed a new optical sensory platform, the phosphor-doped electrospun polymer fibers, for the luminometric detection of common organic solvents, and achieved selective detection of eight different common organic solvents. Moreover, $\mathrm{MeOH}$ and EtOH were successfully distinguished by emission color change response time. We believe that our results provide new possibilities for metal-free organic phosphors to be a novel class of smart optical sensory materials. 


\section{Acknowledgements}

This work was supported by the National Science Foundation (DMREF DMR 1435965) and a Korea Institute of Industrial Technology Grant. The work in Madrid was supported by the Spanish Science Ministry (project MultiCrom, grant no. CTQ2014-58801), and by the Campus of International Excellence (CEI) UAM + CSIC.

\section{Notes and references}

1 (a) M. Irie, T. Fukaminato, T. Sasaki, N. Tamai and T. Kawai, Nature, 2002, 420, 759; (b) T. Mutai, H. Satou and K. Araki, Nat. Mater., 2005, 4, 685; (c) A. Kishimura, T. Yamahita, K. Yamaguchi and T. Aida, Nat. Mater., 2005, 4, 546; (d) Y. Sagara and T. Kato, Nat. Chem., 2009, 1, 605; (e) J. Gierschner and S. Y. Park, J. Mater. Chem. C, 2013, 1, 5818; (f) K. Chung, M. S. Kwon, B. M. Leung, A. G. WongFoy, M. S. Kim, J. Kim, S. Takayama, J. Gierschner, A. J. Matzger and J. Kim, ACS Cent. Sci., 2015, 2, 94; $(g)$ M. S. Kwon, G. Jang, D. Bilby, B. Milián-Medina, J. Gierschner, T. S. Lee and J. Kim, RSC Adv., 2014, 4, 46488.

2 (a) R. Yerushalmi, A. Scherz, M. E. van der Boom and H. Kraatz, J. Mater. Chem., 2005, 15, 4480; (b) Y. Sagara and T. Kato, Nat. Chem., 2009, 1, 605; (c) J. Hu and S. Liu, Macromolecules, 2010, 43, 8315.

3 (a) R. M. Raymo and M. Tomasulo, Chem. Soc. Rev., 2005, 34, 327; (b) M. S. Kwon, J. Gierschner, S. J. Yoon and S. Y. Park, Adv. Mater., 2012, 24, 5487; (c) M. S. Kwon, J. Gierschner, J. Seo and S. Y. Park, J. Mater. Chem. C, 2014, 2, 2552.

4 (a) S. J. Yoon, J. W. Chung, J. Gierschner, K. S. Kim, M. G. Choi, D. Kim and S. Y. Park, J. Am. Chem. Soc., 2010, 132, 13675; (b) Y. Sagara and T. Kato, Angew. Chem., Int. Ed., 2008, 47, 5175; (c) J. Kim, D. T. McQuade, S. K. McHugh and T. M. Swager, Angew. Chem., Int. Ed., 2000, 39, 3868.

5 C. Reichardt, Chem. Rev., 1994, 94, 2319.

6 (a) S. K. Lower and M. A. El-Sayed, Chem. Rev., 1966, 66, 199; (b) C. A. Parker and T. A. Joyce, Chem. Commun., 1968, 749; (c) M. A. El-Sayed, Acc. Chem. Res., 1968, 1, 8; (d) P. Avouris, W. M. Gelbart and M. A. El-Sayed, Chem. Rev., 1977, 77, 793. 7 Y. You and W. Nam, Chem. Soc. Rev., 2012, 41, 7061.

8 (a) G. Zhang, G. M. Palmer, M. W. Dewhirst and C. L. Fraser, Nat. Mater., 2009, 8, 747; (b) P. Lehner, C. Staudinger, S. M. Borisov and I. Klimant, Nat. Commun., 2014, 5, 4460.

9 (a) O. Bolton, K. Lee, H. Kim, K. Lin and J. Kim, Nat. Chem., 2011, 3, 205; (b) D. Lee, O. Bolton, B. C. Kim, J. H. Youk, S. Takayama and J. Kim, J. Am. Chem. Soc., 2013, 135, 6325; (c) M. S. Kwon, D. Lee, S. Seo, J. Jung and J. Kim, Angew. Chem., Int. Ed., 2014, 53, 11177; (d) O. Bolton, D. Lee, J. Jung and J. Kim, Chem. Mater., 2014, 26, 6644; (e) D. Lee, J. Jung, D. Bilby, M. S. Kwon, J. Yun and J. Kim, ACS Appl. Mater. Interfaces, 2015, 7, 2993; (f) M. S. Kwon, Y. Yu, C. Coburn, A. W. Phillips, K. Chung, A. Shanker, J. Jung, G. Kim, K. Pipe, S. R. Forrest, J. H. Youk, J. Gierschner and J. Kim, Nat. Commun., 2015, 6, 8947.

10 (a) M. Bixon and J. Jortner, J. Chem. Phys., 1968, 48, 715; (b) W. Z. Yuan, X. Y. Shen, H. Zhao, J. W. Y. Lam, L. Tang, P. Lu,
C. Wang, Y. Liu, Z. Wang, Q. Zheng, J. Z. Sun, Y. Ma and B. Z. Tang, J. Phys. Chem. C, 2010, 114, 6090; (c) S. Hirata, K. Totani, J. Zhang, T. Yamashita, H. Kaji, S. R. Marder, T. Watanabe and C. Adachi, Adv. Funct. Mater., 2013, 23, 3368; (d) S. Reineke and M. A. Baldo, Sci. Rep., 2013, 4, 3797; (e) J. Xu, A. Takai, Y. Kobayashi and M. Takeuchi, Chem. Commun., 2013, 49, 8447; (f) D. Chaudhuri, E. Sigmund, A. Meyer, L. Rock, P. Klemm, S. Lautenschlager, A. Schmid, S. R. Yost, T. V. Voorhis, S. Bange, S. Hoger and J. M. Lupton, Angew. Chem., Int. Ed., 2013, 52, 13449; (g) M. Koch, K. Perumal, O. Blacque, J. A. Garg, R. Saiganesh, S. Kabilan, K. K. Balasubramanian and K. Venkatesan, Angew. Chem., Int. Ed., 2014, 53, 6378.

11 Limited here to Br6A, since we had shown that G1 had similar photophysical properties in solution.

12 (a) S. J. Strickler and R. A. Berg, Chem. Phys., 1962, 37, 814; (b) J. P. Vikesland and S. J. Strickler, J. Phys. Chem., 1974, 60, 664.

13 According to Strickler-Berg, the rate constant $k_{\mathrm{F}}$ can be calculated from the oscillator strength of the transition responsible for the emission process (in the same solvent with refractive index $n$ ) and the absorption and emission maxima $v_{\mathrm{abs}}, v_{\mathrm{em}}\left(\mathrm{in} \mathrm{cm}^{-1}\right.$ ). In a simplified form this reads: $k_{\mathrm{F}}=0.6671\left[\mathrm{~cm}^{2} \mathrm{~s}^{-1}\right] \frac{\nu_{\mathrm{em}}{ }^{3}}{\nu_{\mathrm{abs}}} n^{2} f$.

14 Since these are very subtle effects, this requires the correct geometry, a TD method to properly describe the energies of allowed and forbidden states, and finally an accurate description of solvent effects. Geometry optimization was carried out with DFT, employing the $\mathrm{BH}$ and HLYP functional with a $6-311 \mathrm{G}^{*}$ basis set, as implemented in the Gaussian09 program package (M. J. Frisch et al., Gaussian, Inc., Wallingford, CT 2009, Gaussian 09, Gaussian, Inc., Wallingford CT, 2009). The functional was shown to properly reproduce torsional angles, which turned out to be the most delicate issue in Br6A with respect to state ordering. Extensive screening of the potential hypersurface gave a planar stable equilibrium geometry (absence of negative frequencies). The TD part was done by employing the B3LYP functional (6-311G* basis set). Solvent effects were taken into account by the implicit polarizable continuum model (PCM).

15 Zero oscillator strength of the $n \pi^{*}$ state is obtained in the calculations; the increase in the experimental value $f=$ 0.053) is ascribed to Herzberg-Teller coupling through outof-plane torsional modes. Indeed, our QC calculations on distorted Br6A structures give considerable oscillator strength to the $\mathrm{n} \pi *$ transition.

16 The qualitative picture does not further change by geometry relaxation, i.e. optimization of the excited states.

17 O. Bolton, The directed heavy atom effect: a design principle for metal-free organic phosphors, Doctoral dissertation, University of Michigan, 2010, pp. 46-47.

18 (a) N. A. Rakow and K. S. Suslick, Nature, 2000, 406, 710; (b) J. Yoon, S. K. Chae and J. M. Kim, J. Am. Chem. Soc., 2007, 129, 3038; (c) S. H. Lim, L. Feng, J. W. Kemling, C. J. Musto and K. S. Suslick, Nat. Chem., 2009, 1, 562.

19 J. Lee, H. T. Chang, H. An, S. Ahn, J. Shim and J. M. Kim, Nat. Commun., 2013, 4, 2461. 